Rev. Inst. Flor. v. 28 n. 1. p. $49-57$ jun. 2016

http://dx.doi.org/10.4322/rif.2016.003

ISSN impresso 0103-2674/on-line 2178-5031

\title{
VARIAÇÃO FENOTÍPICA PARA CARACTERES SILVICULTURAIS EM POPULAÇÕES DE Aspidosperma spp. SEM ESTRUTURA DE PROGÊNIES ${ }^{1}$
}

\section{GENETIC VARIATION FOR SILVICULTURAL TRAITS IN POPULATIONS OF Aspidosperma spp. WITHOUT PROGENY ARRAYS}

\author{
Camila Regina Silva Baleroni RECCO ${ }^{2,7}$; Wanderley dos SANTOS ${ }^{3}$; \\ Danilla Cristina Lemos SOUZA ${ }^{4}$; José CAMBUIM ${ }^{3}$; \\ Marcela Aparecida de MORAES 3 ; Alexandre Marques da SILVA'; \\ Miguel Luiz Menezes FREITAS ${ }^{5}$; Mario Luiz Teixeira de MORAES ${ }^{6}$
}

\begin{abstract}
RESUMO - O objetivo deste estudo foi estimar os parâmetros genéticos para os caracteres silviculturais em Aspidosperma polyneuron Müll.Arg. e Aspidosperma cylindrocarpon Müll.Arg., para fins de conservação ex situ e uso em programas de recuperação ambiental. A coleta de sementes foi feita em árvores de polinização aberta no município de Andradina-SP e o teste experimental foi instalado na Fazenda de Ensino e Pesquisa (FEPE/UNESP), em 1997, com as espécies consorciadas com Inga marginata Willd. (ingá feijão) em linhas alternadas. O delineamento experimental utilizado foi o de blocos casualizados completos com dois tratamentos (Aspidosperma spp.), 32 repetições com parcelas lineares e 4 plantas por parcela. Aos 15 anos de idade foram mensurados diâmetro à altura do peito - DAP, altura de total - ALT e sobrevivência - SOB e os parâmetros genéticos foram estimados pelo programa Selegen. A espécie cylindrocarpon apresentou maior desenvolvimento nos caracteres de crescimento (DAP e ALT) quando comparada com A. polyneuron. A taxa de sobrevivência foi de $71 \%$ demostrando boa adaptabilidade para ambas as espécies ao local de implantação do experimento. A acurácia foi alta, sugerindo maior confiabilidade das análises estatísticas. A herdabilidade foi moderada, indicando considerável controle genético nas espécies quando se observa que o coeficiente de determinação dentro de parcela foi menor que $10 \%$ para ALT e DAP, mostrando que as parcelas lineares são homogêneas, ou seja, o delineamento experimental adotado foi eficiente. Portanto, a ausência do uso de estrutura de progênies nas populações de Aspidosperma spp. estudadas não impossibilitou que fossem obtidas informações relevantes sobre as populações naturais de Aspidosperma, porém vale ressaltar que é de suma importância que novas coletas de sementes sejam realizadas para um estudo com base em teste de progênies.
\end{abstract}

Palavras-chave: conservação genética; parâmetros genéticos; peroba-rosa; peroba-poca; recuperação ambiental.

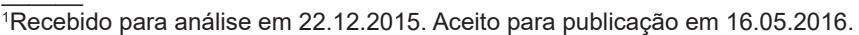

${ }^{2}$ Fundação Educacional de Andradina - FEA, R. Amazonas, 571, Bairro Stella Maris, 16901-160 Andradina, SP, Brasil.

3Pós-graduando em Agronomia, Faculdade de Engenharia de Ilha Solteira, Universidade Estadual Paulista "Júlio Mesquita Filho" - UNESP, Av. Brasil Centro, 56, Caixa Postal 31, 15385-000 Ilha Solteira, SP, Brasil.

${ }^{4}$ Doutorando Ciência Florestal, Faculdade de Ciências Agronômicas Campus de Botucatu, Botucatu, SP, Brasil.

${ }^{5}$ Instituto Florestal, Rua do Horto, 931, 02377-000, São Paulo, SP, Brasil.

${ }^{6}$ Faculdade de Engenharia de Ilha Solteira, Universidade Estadual Paulista "Júlio Mesquita Filho" - UNESP, Departamento de Fitotecnia, Tecnologia de

Alimento e Sócio Economia, Av. Brasil Centro, 56, Caixa Postal 31, 15385-000 Ilha Solteira, SP, Brasil.

${ }^{7}$ Autor para correspondência: Camila Regina Silva Baleroni Recco - milbaleroni@ig.com.br
} 


\begin{abstract}
This study aimed to estimate genetic parameters for the silvicultural traits: diameter at breast height, total height and survival in Aspidosperma polyneuron Müll.Arg. and Aspidosperma cylindrocarpon Müll.Arg., for ex situ conservation and forest restoration purposes. Open pollinated seeds of both species were obtained from natural populations in Andradina-SP, Brazil. The provenance test was installed at Fazenda de Ensino e Pesquisa FEPE/UNESP, in 1997, with the species of mahogany intercropped with Inga marginata Willd. (ingá feijão), in alternate rows. The experimental design was randomization block with two treatments (species of Aspidosperma), 32 replications and four plants per plot in linear plots. Diameter breast height - DBH, plant height $-\mathrm{H}$ and survival $-\mathrm{S}$ were measured at 15 years of age and genetic parameters were estimated by SELEGEN program. The species A. cylindrocarpon showed greater development in growth characters (DBH and $\mathrm{H}$ ) compared to A. polyneuron. The survival rate was $71 \%$ showing good adaptability for both species to the place of experiment implementation. The accuracy was high, suggesting greater reliability of statistical analysis. Heritability was moderate, indicating considerable genetic control in the species when it is observed that the coefficient of determination within plot was less than $10 \%$ for $\mathrm{H}$ and $\mathrm{DBH}$, showing that the linear plots are homogeneous, that is, the experimental design was efficient. Therefore, the absence of the use of progenies structure in the studied populations of Aspidosperma spp. did not preclude that relevant information about the natural populations of Aspidosperma were obtained, but it is noteworthy that it is very important that new seed collections must be carried to a study based on progeny test.
\end{abstract}

Keywords: forest restoration; genetic conservation; native species; peroba-rosa; peroba-poca .

\section{INTRODUÇÃO}

A devastação das florestas brasileiras vem tomando proporções alarmantes em todos os biomas, colocando muitas espécies sob risco de extinção (Mendes e Paula, 2010). Nesse sentido, é fundamental o desenvolvimento de estratégias de conservação genética para garantir a sobrevivência dessas populações, especialmente para as espécies endêmicas.

Nesse sentido, para que uma espécie arbórea tenha condições de sobreviver tanto em circunstâncias de mudanças climáticas como por ação antrópica, deve-se ter uma variação genética substancial para conseguir se adaptar. Desse modo, para garantir a manutenção da variação genética da espécie é necessário estabelecer medidas de conservação. Basicamente, existem duas estratégias de conservação genética, que não são excludentes, mas, sim, complementares: a conservação genética in situ e a ex situ (Kageyama et al., 2001).

$\mathrm{Na}$ conservação in situ as espécies são mantidas em seus habitats e o objetivo é conservar o máximo possível do número de alelos e/ou da diversidade de genótipos. A manutenção da dinâmica nessa estratégia possibilita a evolução contínua de toda a comunidade que vem sendo conservada, incluindo a coevolução entre as plantas, os animais e os microrganismos (Kageyama et al., 2008).
A conservação genética $e x$ situ refere-se àquela em que a espécie é conservada fora de seu ambiente natural. Existem três formas de conservação ex situ: i) conservação de sementes para espécies que produzem sementes ortodoxas; ii) conservação in vitro para espécies que apresentam facilidades na propagação vegetativa e/ou produzem sementes recalcitrantes ou intermediárias; iii) conservação no campo (Valois et al., 2001). Para as espécies arbóreas, o modo mais comum de conservação ex situ é na forma de plantas vivas cultivadas, mais conhecida como formação de população base ou teste de progênies. Normalmente, são constituídas de famílias originárias de polinização livre, dada a facilidade de coleta e instalação do material em condições de campo (Moraes et al., 2006). Esse método é o mais utilizado nas instituições de pesquisa e ensino, como universidades e institutos de pesquisa, que permite estudar a espécie estabelecer um programa de melhoramento e conservação da espécie ao mesmo tempo, e formar futuramente um pomar de sementes melhoradas para suprir a demanda por sementes em uso de restauração florestal, como no presente estudo. 
Nesse sentido, julgou-se oportuno realizar o estudo com duas espécies do gênero Aspidosperma, que estão ameaçadas de extinção devido à degradação de seu habitat natural e sua intensa exploração. Elas se destacam por seu valor madeireiro, que apresenta importância econômica devido ao fornecimento de madeira de excelente qualidade para indústria de móveis, carpintaria e construção civil e naval (Lorenzi, 2002), são também utilizadas na medicina tradicional (Oliveira et al., 2009; Pérez et al., 2012; Ribeiro et al., 2015). Devido a essas peculiaridades, porém associadas à intensa devastação das florestas naturais, as populações de espécies de Aspidosperma encontram-se fragmentadas. Aspidosperma polyneuron, comumente conhecida como peroba-rosa, apresenta-se como uma árvore típica das matas, não existindo em cerrados e cerradões, de ocorrência natural nos estados da Bahia, Espírito Santo, Minas Gerais, Rio de Janeiro, São Paulo, Paraná e Mato Grosso do Sul, e também em países vizinhos, Argentina e Paraguai (Nogueira, 2010). É a espécie de maior valor econômico entre as espécies de Aspidosperma (Carvalho, 2004); devido à sua madeira moderadamente pesada (densidade 0,75 a 0,85 g.cm ${ }^{-3}$ ) (Nogueira, 2010)é indicada para reflorestamentos mistos destinados à recomposição de áreas degradadas de preservação permanente (Lorenzi, 2002). A. polyneuron encontra-se em perigo de extinção (International Unior for Conservation of Nature - IUCN, 2015) e, em virtude de sua importância ecológica, é prioritária sua utilização em programas de conservação de recursos genéticos florestais. A. cylindrocarpon, conhecidapopularmente como peroba-poca, é a espécie mais próxima taxonomicamente de $A$. polyneuron, da qual se separa, sobretudo, pelo menor porte (Rizzini, 1971). É também uma espécie nativa de importância ecológica, utilizada para recuperação de áreas degradadas. Além disso, é empregada em pastos apícolas, como planta ornamental, e sua madeira é utilizada na construção civil (Lorenzi, 2002). Ocorre nos estados de São Paulo, Minas Gerais, Goiás, Mato Grosso e Paraná, muito comum à beira de rios, porém nunca em locais alagados ou brejosos (Nogueira, 2010).
Embora tenha existido uma preocupação em se criar unidades de conservação para conservação in situ das espécies presentes na fauna brasileira em risco de extinção, estas ações, ainda são insuficientes, diante do acelerado processo de degradação das florestas nativas, sendo necessária uma interferência mais direta. Para tanto, algumas instituições de pesquisa têm adotado a conservação ex situ dessas espécies, por meio de testes de procedências e/ou progênies, com o intuito, além da conservação, de subsidiar a coleta de sementes para restauração florestal (Batista et al., 2012; Moraes et al., 2012; Senna et al., 2012; Moraes et al., 2013). Assim, pode-se pensar em estratégias integradas de conservação, associando a conservação in situ, na forma de parques e jardins botânicos, com a conservação ex situ, na forma de armazenamento de sementes, in vitro e in vivo. São de fundamental importância medidas públicas, de incentivo político, para estimular os produtores rurais a também fazerem a conservação genética dessas espécies, em forma de Áreas de Preservação Permanente - APPs e reserva legal.

Pouco se conhece sobre a estrutura genética das espécies de Aspidosperma sp. $\mathrm{Na}$ literatura, a maioria das pesquisas é baseada na caracterização florística e estrutural em fragmentos de ocorrência natural das espécies. Alguns avanços nos estudos de diversidade genética com marcadores moleculares foram contemplados com Torezan et al. (2005), Damasceno et al. (2011), Zimback et al. (2011). Entretanto, trabalhos sobre desenvolvimento e variação genética para os caracteres de crescimento são escassos, sendo o mais recente publicado em 2008 (Sato et al., 2008). Essas informações são preocupantes, as instituições de ensino e pesquisadores deveriam ter mais incentivo para estudar essas espécies, considerando-se sua importância.

Em razão dessa situação, este trabalho foi realizado com o objetivo de conhecer o desenvolvimento e a variabilidade genética de duas espécies de Aspidosperma sp. (Aspidosperma polyneuron e Aspidosperma cylindrocarpon), por meio de caracteres de crescimento, visando à utilização destas espécies para futura formação de um pomar de sementes, de modo a fornecer mudas com qualidade genética para restauração florestal. 


\section{MATERIAL E MÉTODOS}

Sementes foram coletadas de polinização aberta, em 1997, de populações naturais de A. polyneuron Müll.Arg. e A. cylindrocarpon Müll.Arg., na região de Andradina-SP. Na coleta das sementes, procurou-se constituir uma amostra representativa dessas espécies em "bulk" para sua conservação genética ex situ, em função do risco de degradação antrópica ao qual está sujeita. O banco de conservação ex situ foi estabelecido na Fazenda de Ensino Pesquisa e Extensão - FEPE, situada à margem direita do rio Paraná, no município de Selvíria-MS

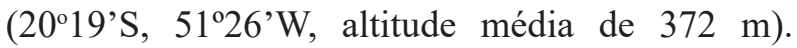
$\mathrm{O}$ relevo da área é moderadamente plano e ondulado. O tipo climático, segundo classificação de Köppen, é do tipo Aw, caracterizado como tropical úmido com estação chuvosa no verão e seca no inverno, temperatura média anual de $24,5{ }^{\circ} \mathrm{C}$, precipitação anual de $1.232 \mathrm{~mm}$ e umidade relativa média anual de 64,8\% (Hernandes et al., 1995). A vegetação original encontrada na área em estudo é típica de Cerrado. O solo local é do tipo Latossolo Vermelho distrófico álico e de textura argilosa (Empresa Brasileira de Pesquisa Agropecuária EMBRAPA, 2006). O teste de procedências foi estabelecido em 11/1997, consorciado em linhas alternadas com Inga marginata Willd. (ingá feijão), espécie classificada como secundária inicial (Instituto de Pesquisas Florestais - IPEF, 1992). O delineamento experimental utilizado foi o de blocos casualizados, com dois tratamentos (espécies de Aspidosperma), 32 repetições e parcelas lineares de quatro plantas por parcelas no espaçamento de $3 \times 3 \mathrm{~m}$. Os caracteres silviculturais avaliados aos 15 anos de idade foram: a) diâmetro à altura do peito - DAP em centímetros; b) altura de plantas - ALT em metros, e c) sobrevivência - SOB em percentagem.

Foram estimados os seguintes parâmetros estatísticos: média ( $\bar{x}$ coeficiente de variação $(C V \%)$, mínimo e máximo; assimetria e curtose, utilizando o programa SELEGEN-REML/BLUP (Resende, 2007). As estimativas de parâmetros genéticos foram calculadas por meio do modelo linear misto, blocos casualizados, para várias espécies sem estrutura de progênies, em um local, segundo o modelo:

$$
\mathrm{y}=\mathbf{X r}+\mathbf{Z g}+\mathbf{W} \mathbf{p}+\mathrm{e} ;
$$

em que: y é o vetor de dados, $r$ é o vetor dos efeitos de repetição (assumidos como fixos) somados à média geral, g é o vetor dos efeitos genotípicos de populações (assumidos como aleatórios), p é o vetor dos efeitos de parcela, $e$ é o vetor de erros ou resíduos (aleatórios). As letras maiúsculas representam as matrizes de incidência para os referidos efeitos. $\boldsymbol{X}, \boldsymbol{Z}$ e $\boldsymbol{W}$ são as matrizes de incidência dos referidos efeitos (modelo 24 do programa SELEGEN) (Resende, 2007).

Os parâmetros genéticos estimados foram: $\hat{\sigma}_{g}^{2}$ variância genotípica entre espécies; $\hat{\sigma}_{c}^{2} \quad$ variância ambiental entre parcelas; $\hat{\sigma}_{e}^{2}$ variância do residual; $\hat{\sigma}_{f}^{2}$ variância fenotípica; $\hat{h}_{g}^{2}$ herdabilidade individual no sentido amplo; $\hat{C}_{p}^{2}$ coeficiente de determinação dos efeitos de parcelas; $r_{\hat{a} a}$ acurácia; $\hat{m}$ média geral.

A correlação fenotípica $\hat{r}_{f}$ entre os dois caracteres foi estimada por:

$$
\hat{r}_{f}=\frac{C \hat{O} V_{f(x, y)}}{\sqrt{\hat{\sigma}_{f(x)}^{2} \cdot \hat{\sigma}_{f(y)}^{2}}}
$$

Em que: $C \hat{O} V_{f(x, y)}$ é a covariância fenotípica aditiva entre os dois caracteres $(x$ e $y), \hat{\sigma}_{f(x)}$ é o componente de variância genética aditiva para $0 x$, e $\hat{\sigma}_{f(\mathrm{y})}$ é o componente de variância genética aditiva para o $y$. As correlações genéticas foram obtidas pelo método REML/BLUP (máxima verossimilhança restrita/melhor predição linear não viciada). $O$ erro padrão para correlações genéticas e fenotípicas foram estimadas por meio de teste $t$, descrito por Bussab e Moretin (2002).

$$
t=\frac{\hat{r}_{p}}{\sqrt{1-\hat{r}_{p}^{2}}} \sqrt{n-2}
$$

Em que: $\hat{r}_{p}$ correlações genéticas ou fenotípicas, número de observações. 


\section{RESULTADOS E DISCUSSÃO}

O valor médio obtido em ALT para A. cylindrocarpon foi de $8,55 \mathrm{~m}$, variando entre indivíduos de $1,7 \mathrm{~m}$ a $17,7 \mathrm{~m}$, o que proporcionou variação de $41,0 \%$. Para $A$. polyneuron, foi observado crescimento inferior, a média foi de $4,50 \mathrm{~m}$, com indivíduos com performance entre $2,0 \mathrm{~m}$ a $8,6 \mathrm{~m}$, e variação dentro da espécie de $29,0 \%$, o que pode ser atribuído às características de crescimento lento em espécies pertencentes ao grupo ecológico das secundárias tardias. Os valores altos do coeficiente de variação podem estar relacionados a não uniformidade das árvores dentro de parcelas, pois, em várias parcelas foram encontradas árvores dominadas, ou seja, as árvores que se encontravam sob o dossel das árvores maiores estão sentindo o efeito de competição por luz. Esses indivíduos dominados apresentam menor desenvolvimento, seu crescimento se torna limitado e há grande variação entre árvores de uma mesma espécie (Barros et al., 2010). Outro fator interessante foi que o local desse experimento é ao lado de uma mata ciliar, ou seja, no estágio inicial as mudas estavem sombreadas, entretanto a espécie Inga marginata teve desenvolvimento rápido, sendo mais dominante que aquelas de Aspidosperma spp. Assim, embora as parcelas fossem lineares, a competição de luz, nutrientes e espaço foi intensa para as três espécies no local.
O valor observado para a média do caráter DAP em A. cylindrocarpon foi de $6,61 \mathrm{~cm}$, com mínimo de 1,34 e máximo de $15,41 \mathrm{~cm}$. O coeficiente de variação foi de $47,5 \%$, próximo ao observado para o caráter ALT. O valor médio obtido para DAP para a espécie $A$. polyneuron foi inferior $(3,60 \mathrm{~cm})$ ao obtido na espécie A. cylindrocarpon, com crescimento superior de 54\% para esta última. O DAP em A. polyneuron variou de 1,34 a $15,41 \mathrm{~cm}$, e uma variação experimental de $47,54 \%$.

Conforme visto na Tabela $1, A$. cylindrocarpon apresentou maior crescimento, com incremento médio anual - IMA de $0,57 \mathrm{~m} /$ ano em ALT e $0,44 \mathrm{~cm} /$ ano de DAP. A. polyneuron apresentou praticamente metade dos valores do IMA da outra espécie, com 0,30 $\mathrm{m} /$ ano para ALT e $0,24 \mathrm{~cm} /$ ano para DAP. Esses valores estão de acordo com Sato et al. (2008), que encontraram $13,14 \mathrm{~cm}$ de DAP (IMA de $0,31 \mathrm{~cm}$ ) e $16,96 \mathrm{~m}$ de ALT (IMA de $0,40 \mathrm{~m}$ ) em $A$. polyneuron aos 42 anos de idade. No geral, ambas as espécies apresentaram baixo desenvolvimento quando comparadas com algumas espécies na literatura avaliadas em idades parecidas, como, por exemplo, Cariniana legalis com 17 anos que apresentou altura de 12,3 m e 13,5 cm de DAP (Sebbenn et al,. 2001) e Myracrodruon urundeuva com 17 anos, 11,5 m de altura e 13,1 cm de DAP (Freitas et al,. 2007).

Tabela 1. Média, coeficientes de variação $(C V(\%))$, valores máximos e mínimos para caracteres silviculturais em espécies de Aspidosperma cylindrocarpon e Aspidosperma polyneuron, aos 15 anos de idade, em Selvíria-MS, Brasil.

Table 1. Mean, coefficient of variation $(C V(\%))$, maximum and minimum values for silvicultural traits in Aspidosperma cylindrocarpon and Aspidosperma polyneuron, at the age of 15 in Selvíria-MS, Brazil.

\begin{tabular}{lcccc}
\hline Parâmetros & \multicolumn{2}{c}{ Aspidosperma cylindrocarpon } & \multicolumn{2}{c}{ Aspidosperma polyneuron } \\
& ALT $(\mathrm{m})$ & DAP $(\mathrm{cm})$ & ALT $(\mathrm{m})$ & DAP $(\mathrm{cm})$ \\
\hline Média & 8,55 & 6,61 & 4,49 & 3,6 \\
$C V(\%)$ & 41,03 & 47,54 & 29,03 & 45,05 \\
Máximo & 17,7 & 15,41 & 8,6 & 9,23 \\
Mínimo & 1,7 & 1,34 & 2 & 0,95 \\
Assimetria & 0,31 & 0,72 & 1,08 & $1,38^{*}$ \\
Curtose & $-0,39$ & 0,35 & 1,24 & $2,50^{*}$ \\
Inferência & Simétrica & Assimétrica Positiva & Assimétrica Positiva & Assimétrica Positiva \\
& Mesocúrtica & Mesocúrtica & Leptocúrtica & Leptocúrtica \\
\hline
\end{tabular}

*Significativo a $5 \%(6,63)$, com um grau de liberdade; ** significativo a $1 \%(3,8)$, com um grau de liberdade pelo teste t.

*Significant at $5 \%(6.63)$ with one degree of freedom; $* *$ significant at $1 \%(3.8)$ with one degree of freedom by $t$ test. 
A classificação das frequências dos indivíduos em relação à distribuição de classes DAP foi diferente estatisticamente pelo teste $t$ a nível de significância de 5\% apenas para a espécie A. polyneuron. A curtose foi classificada como assimétrica positiva leptocúrtica, ou seja, ocorre uma distribuição assimétrica nos valores de DAP com a cauda da curva da distribuição de frequência declinada para a direita. Nesse caso, predominam na amostra valores inferiores à média. A distribuição das frequências apresentou uma distribuição leptocúrtica, ou seja, indica que a distribuição das frequências do DAP é mais estreita do que a curva normal, apresentando menor amplitude dos dados.
A correlação fenotípica para os caracteres ALT e DAP foi de baixa magnitude e não significativo para a espécie $A$. cylindrocarpon $(0,07) \mathrm{e}$ $A$. polyneuron $(0,05)$. Desse modo, os ganhos na associação desses caracteres são independentes e devem ser praticados separadamente.

O valor da taxa de sobrevivência de $71 \%$ revelou boa adaptabilidade de ambas as espécies ao local de implantação do experimento. Por meio da análise de deviance, observaram-se diferenças significativas entre plantas para os caracteres DAP, ALT e SOB $(\mathrm{p}<0,01)$ (Tabela 2).

Tabela 2. Parâmetros genéticos estimados para Aspidosperma cylindrocarpon e Aspidosperma polyneuron, aos 15 anos de idade, em Selviria-MS, para os caracteres altura de planta (ALT-m), diâmetro à altura do peito (DAP-cm) e sobrevivência (SOB-\%).

Table 2. Estimation of genetic parameters of Aspidosperma cylindrocarpon and Aspidosperma polyneuron, at the age 15 in Selvíria-MS, for the characters: plant height (H-m), breast height diameter (DBH-cm) and survival (S-\%).

\begin{tabular}{cccc}
\hline Parâmetros & ALT $(\mathrm{m})$ & DAP $(\mathrm{cm})$ & SOB (\%) \\
\hline$\hat{\sigma}_{g}^{2}$ & 8,1950 & 4,8305 & 0,1186 \\
$\hat{\sigma}_{c}^{2}$ & 0,1354 & 0,3489 & 0,0544 \\
$\hat{\sigma}_{e}^{2}$ & 7,8188 & 5,3579 & 0,0924 \\
$\hat{\sigma}_{f}^{2}$ & 16,1494 & 10,5374 & 0,2655 \\
$\hat{h}_{g}^{2}$ & $0,50( \pm 0,14)$ & $0,45( \pm 0,12)$ & $0,44( \pm 0,11)$ \\
$\hat{C}_{p}^{2}$ & 0,0083 & 0,0331 & 0,2050 \\
$r_{\hat{a} a}$ & 0,9417 & 0,9226 & 0,8681 \\
$\hat{m}$ & 6,5384 & 5,0628 & 0,7148 \\
LRT $\left(\chi^{2}\right)$ & $46,53 *$ & $47,46^{*}$ & $32,17 *$ \\
\hline
\end{tabular}

LRT: Teste da razão de verossimilhança; $\chi^{2}$ qui-quadrado da deviance, *significativo a $1 \%(6,63)$, com um grau de liberdade; $\hat{\sigma}_{g}^{2}$ variância genotípica entre espécies; $\hat{\sigma}_{c}^{2}$ variância ambiental entre parcelas; $\hat{\sigma}_{e}^{2}$ variância do residual; $\hat{\sigma}_{f}^{2}$ variância fenotípica; $\hat{h}_{g}^{2}$ herdabilidade individual no sentido amplo; $\hat{C}_{p}^{2}$ coeficiente de determinação dos efeitos de parcelas; $r_{a ̂ a}$ acurácia; $\hat{m}$ média geral.

LRT: Likelihood ratio test; $\chi^{2}$ chi-square deviance, *significant at $1 \%$ (6.63) with one degree of freedom; $\hat{\sigma}_{g}^{2}$ genotypic variance among species; $\hat{\sigma}_{c}^{2}$ environmental variance between plots; $\hat{\sigma}_{e}^{2}$ the residual variance; $\hat{\sigma}_{f}^{2}$ phenotypic variance; $\hat{h}_{g}^{2}$ individual heritability in the broad sense; $\hat{C}_{p}^{2}$ coefficient determining plots effects; $r_{\hat{a} a}$ accuracy; $\hat{m}$ average.

Os valores dos coeficientes de determinação dos efeitos de parcela $\left(\hat{C}_{p}^{2}\right)$ foram baixos para os dois caracteres em estudo e paras as duas espécies, o que indica que não houve influência da distribuição das parcelas dentro das repetições

na estimativa dos parâmetros genéticos, o que determina a homogeneidade do ambiente na área experimental. De acordo com Resende (2002), uma estimativa de até $10 \%$ não chega a interferir na estimativa dos parâmetros genéticos. 
Mesmo que os coeficientes de variação experimental tenham sido elevados (Tabela 1), não se pode afirmar que as estimativas sejam acuradas. Resende (2002) afirma que uma estatística adequada para avaliar a qualidade dos experimentos deveria levar em consideração o coeficiente de variação experimental (CVe), o número de repetições e o coeficiente de variação genética. Esse conjunto de parâmetros determina a acurácia seletiva ( $\mathrm{raa}_{\mathrm{a}}$. Para Resende e Duarte (2007), a acurácia é considerada como de valor alto em no intervalo entre $70 \%$ a $90 \%$, indicando alta precisão no acesso à variação genética verdadeira a partir da variação fenotípica observada nos caracteres, no presente estudo o valor da acurácia para os caracteres avaliados está dentro deste intervalo (Tabela 2). Segundo Vleck et al. (1987), os valores preditos permitem indicar com mais segurança se o germoplasma em estudo maximizará as possibilidades de progresso genético com seleção. A literatura prática pertinente ao assunto, apresentada por Resende et al. (1995) e Costa et al. (2000), tem demonstrado a importância da acurácia para apontar o grau de confiabilidade dos resultados obtidos na avaliação genética.

Como plantios experimentais semelhantes ao do presente estudo (com espécies nativas e idade estabelecida) são praticamente inexistentes, as informações produzidas aqui são importantes para: a) fornecer informações sobre o comportamento silvicultural de espécies nativas em consórcio a longo prazo; b) sugerir medidas adequadas de manejo, como poda, para promover melhor arquitetura das plantas; c) avaliar a taxa de sobrevivência e adaptação dessas espécies para fins de conservação genética ex situ; d) subsidiar avaliações da variabilidade e controle genético no local, a fim de se estabelecer no futuro a formação de um pomar de sementes melhoradas para suprir o mercado de reflorestamento.

\section{CONCLUSÕES}

Aspidosperma cylindrocarpon apresentou maior crescimento e dominância em relação à A. polyneuron aos 15 anos.
A ausência do uso de estrutura de progênies no experimento não impossibilitou a obtenção de informações relevantes sobre a diversidade genética do plantio em duas espécies de Aspidosperma, porém, vale ressaltar que é de suma importância para o estudo de populações que novas coletas de sementes sejam realizadas para um estudo de progênies.

\section{REFERÊNCIAS BIBLIOGRÁFICAS}

BARROS, B.C. et al. Volumetria e sobrevivência de espécies nativas e exóticas no pólo gesseiro do Araripe, PE. Revista Ciência Florestal, v. 20, n. 4, p. 641-647, 2010.

BATISTA, C.M. et al. Estimativas de parâmetros genéticos e a variabilidade em procedências e progênies de Handroanthus vellosoi. Pesquisa Florestal Brasileira, v. 32, n. 71, p. 269-276, 2012.

BUSSAB, W.O.; MORETIN, P.A. Estatística básica. 5. ed. São Paulo: Saraiva, 2002.

CARVALHO, P.E.R. Peroba-rosa - Aspidosperma polyneuron. Colombo: Embrapa Florestas, 2004. 12 p. (Circular Técnica, 96).

COSTA, R.B. et al. Selection and genetic gain in populations of Hevea brasiliensis with a mixed mating system. Genetics and Molecular Biology, v. 23, p.671-679, 2000.

DAMASCENO, J.O. et al. Genetic differentiation in Aspidosperma polyneuron (Apocynaceae) over a short geographic distance as assessed by AFLP markers. Genetics and Molecular Research, v. 10, n. 2, p. 1180-1187, 2011.

EMPRESA BRASILEIRA DE PESQUISA AGROPECUÁRIA - EMBRAPA. Centro Nacional de Pesquisa do Solo. Sistema brasileiro de classificação de solos. 2. ed. Rio de Janeiro: Embrapa Solos, 2006. 306 p.

FREITAS, M.L.M. et al. Formação de pomar de sementes a partir da seleção dentro de teste progênies de Myracrodruon urundeuva. Rev. Inst. Flor., v. 19, n. 2, p. 65-72, 2007. 
HERNANDES, F.B.T.; LEMOS-FILHO, M.A.F; BUZETTI, S. Software HIBRISA e o balanço hídrico de Ilha Solteira. Ilha Solteira: UNESP, 1995. p. 45.

INSTITUTO DE PESQUISAS E ESTUDOS FLORESTAIS - IPEF. Recomposição da vegetação com espécies arbóreas nativas em reservatórios de usinas hidrelétricas da CESP, Série Técnica IPEF, v. 8, n. 25, p.1-43, 1992.

INTERNATIONAL UNION

FOR CONSERVATION OF NATURE - IUCN. The IUCN Red List of Threatened Species. Version 2014.3. Disponível em: <www.iucnredlist.org $>$. Acesso em: 11 Feb. 2015.

KAGEYAMA, P.Y.; GANDARA, FB.; VENCOVSKY, R. Conservação in situ de espécies arbóreas tropicais. In: NASS, L.L. et al. Recursos genéticos e melhoramentos de plantas. Rondonópolis: Fundação MT, 2001. p. 149-158.

Conservação in situ de espécies arbóreas tropicais. In: NASS, L.L.; VALOIS, A.C.C.; MELO, I.S. Recursos genéticos vegetais. Brasília, DF: Embrapa Recursos Genéticos e Biotecnologia, 2008.

LORENZI, H. Árvores brasileiras. 4. ed. Nova Odessa: Instituto Plantarum. v. 2, 368 p.

MENDES, H.S.J.; PAULA, D.R. Conservação genética de espécies arbóreas florestais. Cultivar Grandes Culturas, 2010. Disponível em: <http://www.grupocultivar.com. br/artigos/conservacao-genetica-de-especies-arboreasflorestais $>$. Acesso em: ago. 2015.

MORAES, M.A. et al. Estimating coancestry within open-pollinated progenies of a dioecious species: the case study of Myracrodruon urundeuva. Silvae Genetica, v. 61, p. 256-264, 2012.

et al. Variação genética em progênies de Jacaranda cuspidifolia Mart. utilizando o delineamento sistemático tipo "leque". Scientia Forestalis, v. 41, n. 98, p. 175-183, 2013.

MORAES, M.L.T.; MORI, E.S.; RODRIGUES, C.J. Delineamento de pomares multiespécies. In: HIGA, A.R.; SILVA, L.D. Pomar de sementes de espécies florestais nativas. Curitiba: FUPEF, 2006. $264 \mathrm{p}$.
NOGUEIRA, J.C.B. Reflorestamento misto com essências nativas: a mata ciliar. São Paulo: Instituto Florestal, 2010. p.148.

OLIVEIRA, V.B. et al. Atividade biológica e alcaloides indólicos do gênero Aspidosperma (Apocynaceae): uma revisão. Revista Brasileira de Plantas Medicinais, v. 11, p. 92-99, 2009.

PÉREZ, N.M.; TORRICO, F.B.; MORALES, A. Acute toxicity, antinociceptive activity and indole alkaloids of aqueous extract from bark of Aspidosperma cuspa (Kunth) Blake. Journal of Ethnopharmacology, v. 143, n. 2, p. 599-603, 2012.

RESENDE, M.D.V. Genética biométrica e estatística no melhoramento de plantas perenes. Brasília, DF: EMBRAPA Informação Tecnológica, 2002. p. 975.

SELEGEN - REML/BLUP: sistema estatístico e seleção genética computadorizada via modelos linear misto. Colombo: Embrapa Floresta, 2007. p. 359.

et al. Acurácia seletiva, intervalos de confiança e variância de ganhos genéticos associados a 22 métodos de seleção em Pinus caribaea var. hondurensis. Revista Floresta, v. 24, n. 1/2, p. 35-45, 1995.

DUARTE, J.B. Precisão e controle experimental de qualidade em experimentos de avaliação de cultivares. Pesquisa Agropecuária Tropical, v. 37, n. 3, p. 182-194, 2007.

RIBEIRO, E.F. et al. Diuretic effects and urinary electrolyte excretion induced by Aspidosperma subincanum Mart. and the involvement of prostaglandins in such effects. Journal of Ethnopharmacology, v. 163, p. 142-148, 2015.

RIZZINI, C.T. Árvores e madeiras úteis do Brasil: manual de dendrologia brasileira. São Paulo: Edgard Blücher, 1971. p. 294.

SATO, A.S. et al. Crescimento e sobrevivência de duas procedências de Aspidosperma polyneuron em plantios experimentais em Bauru, SP. Rev. Inst. Flor., v. 20, n. 1, p. 23-32, 2008. 
RECCO, C.R.S.B. et al. Variação fenotípica em Aspidosperma spp.

SEBBENN, A.M. et al. Depressão por endogamia em populações de jequitibá-rosa. Rev. Inst. Flor., v. 13, n. 1, p. 61-81, 2001.

SENNA, S.N. et al. Variação e parâmetros genéticos em teste de progênies de polinização livre de Peltophorum dubium (Sprengel) Taubert em Luiz Antonio -SP. Scientia Forestalis, v. 40, n. 95, p. 345-352, 2012.

TOREZAN, J.M.D. et al. Genetic variability of pre and post-fragmentation cohorts of Aspidosperma polyneuron Muell. Arg. (Apocynaceae). Brazilian Archives of Biology and Technology, v. 48, n. 2, p. 171-180, 2005.

VALOIS, A.C.; NASS, L.L.; GOES, M. Conservação ex situ de recursos genéticos vegetais. In: NASS, L.L. et al. Recursos genéticos e melhoramento: plantas. Rondonópolis: Fundação MT, 2001. p. 29-55.

VLECK, L.D. van; POLLAK, E.J.; OLTEMACU, E.A.B. Genetics for the animal sciences. New York :W.H. Freeman, 1987. p. 391.

ZIMBACK, L. et al. Estrutura genética de peroba (Aspidosperma polyneuron) no Estado de São Paulo, Brasil. Rev. Inst. Flor., v. 23, n. 2, p. 265-277, 2011. 